\title{
Difficulties Facing the Patients and Extra Burden for Healthcare Providers by Non-adherence to the Medications - A Comprehensive Review
}

\author{
Yahaya Hassan', Ahmed Alaa Al-Temimi², Rizwan Ramli³, Mohd Firdaus M. Saad ${ }^{3}$, Noorizan A. Aziz' \\ ${ }^{1}$ Clinical Pharmacy Unit, School of Pharmacy, Management and Science University, Shah Alam, Selangor, MALAYSIA. \\ ${ }^{2}$ Clinical Pharmacy Department, School of Pharmacy, Monash University Malaysia, Sunway, Selangor, MALAYSIA. \\ ${ }^{3}$ Department Pharmacy Practice, Universiti Teknologi Mara, Puncak Alam, Selangor, MALAYSIA.
}

\begin{abstract}
Background: Compliance is commonly and always being confused with concordance, which is a process by which a patient and health care provider make discussion and decisions together about treatment and their outcomes. General worldwide, noncompliance is a major obstacle and main challenges to the effective and efficient health care delivery. Estimation in 2003 by the World Health Organization indicates that only approximately $50 \%$ of chronic diseases patients that us living in developed countries follow their treatment as scheduled and recommended by clinicians. Objectives: To report on the status and future trends of Medications adherence. Materials: Published journal article and conference reports. Method: Data Extraction, data on area related to the patients and healthcare providers for non-adherence to the Medications were gleaned from various publications. Results and Conclusion: Low compliance to prescribed medical is a complex problem, especially for patients with a chronic illness. With increasing numbers of medications shown to do better than harm when taken as prescribed, low compliance is a growing concern, seriously undermining the benefits of current medical care. Medical non-compliance has been identified as a major public health problem that imposes a considerable financial burden upon modern health care systems.
\end{abstract}

Key words: Non-adherence, Quality in health care, Patient burden, Drug Compliance, Comprehensive.

\section{INTRODUCTION}

Compliance is commonly and always being confused with concordance, which is a process by which a patient and health care provider make discussion and decisions together about treatment and their outcomes. General worldwide, non-compliance is a major obstacle and main challenges to the effective and efficient health care delivery. Estimation in 2003 by the World Health Organization indicates that only approximately $50 \%$ of chronic diseases patients that us living in developed countries follow their treatment as scheduled and recommended by clinicians.

Low compliance to prescribed medical is a complex problem, especially for patients with a chronic illness. With increasing num- bers of medications shown to do more good than harm when taken as prescribed, low compliance is a growing concern, seriously undermining the benefits of current medical care. Medical non-compliance has been identified as a major public health problem that imposes a considerable financial burden upon modern health care systems. ${ }^{1}$

In particular for example, low rates of adherence to therapies for asthma, diabetes and hypertension are thought to contribute substantially to the human and economic burden of those conditions as stated above. Compliance rates may be overestimated in the medical article, as compliance is often high in the setting of a formal clinical trial
Submission Date: 15-10-2018; Revision Date: 19-05-2019; Accepted Date: 12-08-2019

DOI: 10.5530/ijper.53.4s.143 Correspondence:

Dr. Yahaya Hassan, Professor, Clinical Pharmacy Unit, Management and Science University, Shah Alam, Selangor, MALAYSIA.

Phone no: +60 195515611 Email id: profyahaya@gmail. com

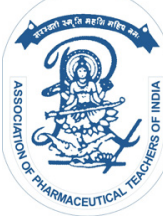

www.ijper.org 
or in the theory on paper but drops off when it comes to the practicality or in a "real-world" setting.

In health field, taking medications prescribed by patients is critical since it will be denoting the level of understanding of their diseases as well as medications. The term adherence or medication adherence always refers patients whether they take their medications as ordered by medical officer, specialist or consultant. (e.g., twice daily, one month), it is also defined as whether they are able to take medications consistently and continuously as prescribed by doctor as well as counselled by pharmacist regarding the usage of medications prescribed. As a pharmacist in government hospital, we have to exercise on the empowerment of the patients' medication adherence. ${ }^{2}$

There are five rights of a patient that needs to be informed by health care provider especially pharmacist on duty. In order to increase adherence to medication, patient should know their correct name of medication taken to ensure they are appreciating of it. Secondly, the pharmacist should ensure the right dose given to the patient with the correct frequency and the time of administration. Non-adherence to medication hence is a significant concern to clinicians, healthcare systems and other stakeholders (e.g., payers) because of increasing evidence that it is prevalent and associated with adverse outcomes and higher costs of care. Until now, measurement of patients' adherence towards medication and use of interventions to enhance medication adherence are negligible in routine clinical practice especially in the hospital setting.

Adherence also sometimes may be defined as the "proactive, voluntary and collaborative participation of our patient in a mutual way and they are always acceptable course of behaviour to give a therapeutic outcome in the treatment. This definition explains that the patient has a right and that both patients and health care providers mutually establish and discuss treatment goals and outcomes and the best and appropriate medical regimen. Medication adherence hence usually refers to whether patients take the drugs as prescribed, as well as whether they are taking it continuously to take a prescribed medication. Furthermore, medication compliance behaviour has thus been categorized under two main concepts, which are, adherence and persistence. Although conceptually similar each other, adherence originally refers to the intensity of medications use during the length of therapy, whereas persistence refers to the general overview or overall duration of drug treatment. ${ }^{3}$

The WHO Adherence meeting in June 2001 concluded that defining adherence as "the extent and degree to which the patient follows medical instructions" was a helpful and precious starting point. However, the word "medical" was felt to be insufficient in describing the range of interventions used to treat chronic illnesses. Furthermore, the term "instructions" suggests that the patient is a passive, accepting recipient of expert advice as opposed to an active collaborator in the treatment process. It was recognized that adherence to any regimen of the treatment reflects behaviour of one type or another.

Later, there are lot of efforts to improve and enhance medication compliance have been targeted like simplifying packaging of medication, providing and emphasizing the effective medication reminders, improving patient education towards therapy and limiting the number of medications prescribed simultaneously based on the best choice of patient background and their health status.

In other definition, medication compliance refers to the patients' act of conforming and attaching to the recommendations plan that made by the provider with respect to timing and duration, dosage and frequency of medication taking.

Furthermore, the Medication Possession Ratio (MPR) is operationalized in retrospective assessments as the number of doses dispensed in relation to the dispensing period. Retrospective prescription of a patient claims database analyses lack and insufficient the details of daily dosing that are available with prospective electronic monitoring; however, as these tools are often the very important and the only sources available to assessing compliance, it is suggested and recommended that this limitation is noted when describing compliance in these instances. ${ }^{4}$

\section{Prevalence of Non-Adherence Medications among Chronic Illness Patients}

Non-adherence to medications is very common for chronic patients including cardiovascular diseases. After patient with acute myocardial infarction hospitalization, Jackevicius et al. reported that almost one fourth of patients (approximately 24\%) did not even fill and attach to their cardiac medications starting at day 7 post discharge. Among patients discharged with medications like aspirin, statin group and $\beta$-blockers after post-acute myocardial infarction, one study stated that there are about $34 \%$ of patients stopped at least 1 medication and 12\% stopped all 3 medications within 1 month after hospital discharge. This is shows that beyond the early discharge period, there appears to be a progressive decrease in the level of adherence to prescribed cardio protective medications (e.g., statins, 
$\beta$-blockers) over time. Rather than that, Newby et al. found that patient self-report of consistent and continuously use of cardiac medications over 6 to 12 months was very low and declining in trend, with approximately three fourths of patients reporting persistent and compliance to aspirin use (71\%), whereas less than half reported persistent use of $\beta$-blockers $(46 \%)$, followed by lipid-lowering agents (44\%) and all 3 medications (21\%) after confirmed diagnosis of coronary artery disease by coronary angiography result. ${ }^{5}$

Another study demonstrated that about $40 \%$ of patients were still taking statin medications 2 years after warded for their acute coronary syndrome and adherence was even lower for patients taking statins for chronic his or her coronary artery disease. Although the transition period from hospital discharge to the outpatient setting appears to be a particularly critical and high-risk period, nonadherence to medication continues to decline during the long-term follow-up phase for coronary artery patient.

For other cardiovascular conditions, the prevalence of non-adherence medication varies extremely depending on the population patients studied and the specific medications assessed. For example, Vrijens et al. using medication event monitor (MEMS) data, stated that about half of all patients that was prescribed with antihypertensive medications stopped from taking them within one year of the initial phase of therapy. They also found that on any 1 day, patients omitted approximately $10 \%$ of the scheduled doses of medications. In contrast, Bramley et al. found that about $75 \%$ of patients on monotherapy for hypertension disease were highly adherence, denoting as a medication possession ratio of $80 \%$ to $100 \%$. Among heart failure patients, studies of the level medication adherence also found widely differing rates of non-adherence. For example, a study reported persistence rates of $79 \%$ for reninangiotensin inhibitors, $65 \%$ for $\beta$-blockers, $56 \%$ for spironolactone and $83 \%$ for statins 5 years after an index heart failure hospitalization. ${ }^{6}$

In contrast, the rate of nonadherence based on pill counts was much lower in the one randomised study entitled of Candesartan in Heart Failure: Assessment of Reduction in Mortality and Morbidity (CHARM), controlled trial of patients with heart failure, resulted in $11 \%$ of patients taking fewer than $80 \%$ of the prescribed pills. Although non-adherence to medications is prevalent in cardiovascular populations, the variability of the methods of assessment of medication use (e.g., self-report or pharmacy refill data) makes comparisons across studies and across cardiovascular conditions difficult. $^{7}$
The relationship between the patient and the health care provider including physician, nurse, pharmacist or other health practitioner must be a partnership that draws on the abilities of each. The literature has identified and verified that the quality of the treatment relationship as being an important factor to improves the treatment adherence. Effective treatment relationships and rapport are characterized by an atmosphere in which alternative therapeutic means are discovered and explored, the regimen is negotiated, adherence is discussed and follow-up is planned by all health care providers.

\section{Relationship between Medication Adherence and Patient Outcomes}

Many observational studies have been evaluated the relationship between medication adherence and patient outcomes. In general, the studies have focused on medications that have already been resulted in clinical trials to be efficacious and effectiveness therefore are trying to assess the effectiveness of the particular medications in routine clinical daily practice.

Pharmacy refill database and patient self-report are the mostly commonly used as adherence assessment methods in the studies. For example, high adherence (which is defined as medication possession ratio of $80 \%$ to $100 \%$ ) to antihypertensive drugs was associated with significant impact of blood pressure control when compared to patients with medium or low levels of adherence. Besides, each incremental 25\% increase in proportion of days covered for statin medications was resulted with an approximately $3.8-\mathrm{mg} / \mathrm{dL}$ reduction in Low Density Lipoprotein (LDL) cholesterol. ${ }^{8}$

Furthermore, patients that non-adherence to cardiovascular medications has been associated with high risk of morbidity as well as mortality. According to studies the non-adherence to statins in the year after hospitalization for myocardial infarction was related with $12 \%$ to $25 \%$ increased relative hazard for mortality. Other than that, in the chronic coronary artery disease, non-adherence to cardio protective medications such as $\beta$-blockers, statins and/or angiotensin-converting enzyme inhibitors was parallel with about $10 \%$ to $40 \%$ relative increase in risk of cardiovascular warded and a $50 \%$ to $80 \%$ relative increase in risk of mortality of that non-adherence patient. ${ }^{9}$

Another studies showed that patients omitting Clopidogrel $75 \mathrm{mg}$ within 1 month post hospitalization for acute myocardial infarction and drug-eluting stent placement were significantly will have an adverse outcome, thus end up with increasing trend of re-hospitalization and mortality, in the subsequent next 11 months. 
Besides, very weak adherence to heart failure drugs was significantly associated with an increased in the number of cardiovascular-related emergency department visits due to instable condition of that patient. These effectiveness studies supportive the previous data that the benefits of cardiovascular medications in routine clinical practice and highlight the importance of taking these medications as prescribed to optimize patient outcomes hence reducing the hospitalization. ${ }^{10}$

\section{Healthy Adherer Effect}

Although the relation and association between medication nonadherence and adverse outcomes has been showed in many observational studies, some concern has been raised that this association and relation may be, at least in part, closely related to the effect of a "healthy adherer". The healthy adherer effect explains that the lower risk of adverse outcomes associated with adherence may be a temporarily masking and surrogate marker for overall performance of a healthy behaviour. This is supported by analyses of randomized and controlled clinical trials resulted even more adherence to placebo is associated and related with better outcomes and effect than for patients who are non-adherent to active therapy. These findings were first reported in 25 years ago by the Coronary Drug Project, which actively involved Clofibrate. Besides, in the $\beta$-blocker Heart Attack Trial, patient with very poor adherence was linked and associated with higher mortality risk regardless of whether that patients were randomized to antihypertensive propranolol or placebo. ${ }^{9,10}$

Similar findings were showed in the Canadian Amiodarone Myocardial Infarction Arrhythmia Trial (CAMIAT) study on the patient's sudden death, total cardiac mortality and all-cause mortality and in the CHARM program for all-cause mortality. It explains that patients who take their medication regularly as requested by the health care provider are also more likely to perform other healthy behaviours and physical activities, such as eating properly and exercising regularly at least 3 times a week. These behaviours are often miss looked and not measured directly in either prospective or retrospective studies. ${ }^{11}$

However, there is also evidence and proof against the healthy adherer effect because a major factor in observed associations and relation between medication adherence and outcomes. For example, Rasmussen et al. in a study on post-myocardial infarction patients demonstrated that the association between adherence to cardiovascular drugs and outcomes in which neither clinical evidence nor biological plausibility existed was assessed and analysed. Interestingly, they demonstrated that adherence and compliance to cardiovascular medications was not directly associated with hospitalization for lung, prostate, or breast cancer and finally concluded that the benefit of adherence was directly related to the cardiovascular medication rather than other causes like epiphenomenon of a healthy adherer effect as discussed earlier. Although the topic will be continuously debatable, the medications and drugs under that study have often been demonstrated in randomized, controlled clinical trials to be safe, efficacious and therefore, the importance and significant of taking these medications as given should be reinforced and highlighted. ${ }^{12}$

\section{Assessment of Medication Adherence}

Predictors of non-adherence to medication may be useful to identify elderly who are most in need of interventions to improve the adherence. Among the nonadherence warning signs always happened may include:

Not filling a new prescription as scheduled

Not obtaining refills as often as expected for medications taken on a chronic basis especially during given appointment date

Not refilling prescriptions for chronic medications as required

Not completing or omitting the entire course of regime for short-term treatment.

Identification and verification of older persons at risk for non-adherence to medication prescribed is just the first step in addressing this potential problem. In order to give an impact on medication's adherence, health care providers must understand and acknowledge the challenges and barriers to adherence and tools and methods that can be applied to overcome and solve these problems. Hughes et al. 2004 mentioned that overcoming one barrier or providing a single intervention will not guarantee patients medication adherence. In fact, several studies showed that no single intervention is adequate and sufficient to ensure medication adherence. A combination of approaches and methodology with different target tailored to the individual person's needs that target specific barriers to adherence and reinforce positive behaviours is the most effective Krueger et al. 2005.

Adherence to medication therapy has been monitored long time ago since the time of Hippocrates, when the effects of various potions were recorded and properly documented with highlighting notations of whether the patient had taken them or not. Even up today, patients' self-reports can simply and effectively measure on adherence. Furthermore, the methods and tools 
available for measuring adherence can be categorized into direct and indirect methods. Each method has their own advantages and disadvantages and in measuring adherence until now no method is considered the gold standard and validated. Directly observed therapy on how the patients taking their own drugs, measurement of concentrations and volume of a drug or its metabolite in blood or urine and detection or measurement of a biologic marker added to the drug formulation in blood are examples of direct methods and approach of measures of adherence. However, direct approaches are expensive in price, burdensome to the health care provider and susceptible to alteration by the patient themselves. However, for some drugs, this method like measuring these levels is a good and commonly used means of assessing adherence. Nowadays, the serum concentration of anticonvulsant drugs such as phenytoin or valproic acid will probably reflect and effect adherence to regimens with these medications taken and sub-therapeutic levels will probably indicate poor adherence or suboptimal and insufficient dose strengths. ${ }^{12}$

Questioning the patient or by using a questionnaire, patient diaries recorded and clinical response assessment are all methods that are relatively easy and simple to use but questioning the patient on medication intake can be susceptible to misrepresentation and tends to result in the health care provider's overestimating the patient's adherence and it will be led to falseinterpretation. The use of a patient's clinical response as a baseline in measuring is confounded by many factors and causes other than adherence to a medication regimen only that can account for clinical outcome and goal. The most common method used to measure adherence, other than patient questioning, has been pill counts that is counting the number of pills and capsule given that remain in the patient's medication bottles or vials or envelope. Although this technique is looks simplicity and empiric nature of this method are attractive to many investigators, the method is actually subject to many problems, because patients can switch their drugs between bottles or envelope and may discard pills before next visits in order to appear to be following the regimen. ${ }^{13}$

Electronic monitors are able and capable of recording and stamping the time that the bottles is opening, number of dispensing drops left (as in the case of glaucoma) or activating a canister as in the case of asthma on multiple occasions have been used for more than approximately 30 years. ${ }^{14,15}$ Rather than providing weekly or monthly averages, these devices provide sufficient, precise and detailed insights into patients' behaviour towards taking medication, but they are still indirect methods of measuring adherence to medications; they do not document and record whether the patient actually ingested and taking the correct drug prescribed or correct dose. ${ }^{16}$

\section{Causes of Poor Medication Adherence}

Adherence research has unsuccessfully to find clear associations between fixed patient characteristics, like age, gender, or education and adherent or nonadherent status. However, when looking beyond these sociodemographic factors, modifiable patient characteristics and approaches, such as behaviour and attitudes, have been shown to be challenges and barriers to medication adherence. For example, forget to take, feel fear of undesired side effects of medication, reservations toward taking of drug and insufficient educational information and understanding regarding drug management and drug holidays have been found to be associated to patients' medication nonadherence. A report by the WHO, 2016 showed the general importance of improving and enhancing adherence to longterm medical therapy. In the report WHO concluded that adherence to medication is the result of a complex interaction and combination of the social environment background, patients and healthcare professionals. It has been shown that low adherence to long-term medication treatment often occurs when the treatment is very complex or when the disease presence is asymptomatic such as hypertension. Previous studies have shown that poor adherence to too many drugs regimen is due to patient perception and impression that the disease is not significant to them, think of adverse drug effects, lack of treatment efficacy and effectiveness and the patient's poor or incomplete knowledge of the chronic disease. Known factors and barriers can be classified in at least three ways:

1. Health literacy barriers related to not knowing what to do and why to take drug.

2. Personal behavioural barriers that emphasize not having the skills necessary to accomplish and complete medication management in the context of everyday daily life.

3. System or administrative barriers and limitations related to access and fragmentation of care.

Thus, the most effective and efficient way of interventions use and practice is a combination of methodology of approaches and address literacy, behaviour and organizational issues. System and administrative factors are derived from a patient's incapability to afford or difficulty in affording their drugs and may include, for example, lack of adequate and inability healthcare 
coverage or insurance, unemployment, retirement patient and indigence. ${ }^{17}$

The most common reasons given by patients for not taking their medications are forgetfulness about $30 \%$, other priorities approximately $16 \%$, deciding to skip or omit a dose about $11 \%$, lack of updated information about $9 \%$ and emotional disturbances for about $7 \%$ and about $27 \%$ of patients give no reason possibility due to negligence. Factors that are related negatively with adherence include increased complexity or duration of a medication regimen and therapy, think of side effects, very old age factor, extreme poverty, having social isolation and psychiatric disorders, especially paranoid; a study on adherence in HIV-positive individuals, which included these patient-level factors, found that older age of HIV-positive patients with neurocognitive impairments or medication problems were at an increased risk of sub-therapeutic and suboptimal medication adherence of patient when compared to their younger counterparts. ${ }^{18}$

Perhaps because patients take and use the medications by themselves, most of the attention on medication nonadherence focuses and highlighting on the patients themselves; however, Tarn et al. suggest that physician approaches and behaviours may critically and significantly contribute to medication nonadherence. It is not only is the ability of physicians to recognize and trace non-adherence poor, they always lack adequate training and time to alleviate patients 'problems. Tarn et al. stated major weakness and deficits in the information and education that physicians deals and given to patients when prescribing and counselling new medications. The data on a study showed that in over $65 \%$ of all cases, at least one critical piece of information and education was not provided by health care providers. Barriers and limitation to adherence may also be related and associated to poor rapport and interactions and/ or communication between patients, healthcare providers and the healthcare system itself. Traditionally, physicians and designated healthcare workers have played an important and key role in achieving medications adherence by providing patients with the reason and rationale for treatment and involving family, relatives and caregivers whenever needed however, it is time and resource intensive for health care providers to evaluate and assess and influence adherence alone on a timely and ongoing basis. $^{19}$

For many patients, the cost of medication itself directly affects and influences theirlevel of medication adherence. For example, in the USA the coverage disparities and gap in Part D for patients on Medicare may be a financial challenge, especially for expensive medications; however, sometimes generic alternatives provided at lower and minimum cost can provide a solution. Additionally, several studies have stated a strong and consistent relationship and association between co-payments and medication adherence. A substantial portion and division of medication nonadherence is driven by out-of-pocket costs of multiple medications. Patients with severe chronic illness are likely to skip or discontinue their medications in response to burden co-paymentincreases. Unfortunately, those with multiple chronic conditions and presumably using more medications are more susceptible to cost-related nonadherence, which may exacerbate the severity of chronic conditions, generate adverse health events reaction and increase healthcare use. Payers could selectively reduce or eliminate co-payments for highly beneficial drugs for some patient subgroups, such as statins group in patients with heart disease or diabetes or blood pressure-reducing medications for those with hypertension. This has led to a wave movement, known as "value-based insurance design," to reduce co-payments for the most effective, high value medications. An Observational studies have shown that lowering and reducing co-payments for highly effective chronic therapies can greatly improve adherence.

Age by itself is not confirm determining factor contribute to medication non-adherence. Rather, there are many factors and causes that may combine to render older persons less able and sensitive to adhere to their medication regimens. However, there is evidence and facts to suggest that with the proper highly motivation, education and support by both parties, older persons can overcome many barriers to medication adherence (US Department of Health and Human Services, 1990). According to World Health Organization Adherence is a multidimensional phenomenon that can be determined by the interplay of five sets of factors, termed "dimensions" by:

- Social and economic factors

- Provider-patient and health care system factors

- Patient's condition-related factors

- Patient's therapy-related factors

- Patient-related factors

Patient-related factors are just one causes and determinant of adherence behaviour (World Health Organization, 2003). The common belief that a person is solely responsible for using and taking their medications often indicates and reflects a misunderstanding of how other factors affect people's medication-taking behaviour and their capacity to adhere to treatment regimens. ${ }^{20}$ It is clear that adherence is a complex behav- 
ioural process strongly influenced by the combination of several factors like environments in which people live, health care providers practice and health care systems deliver care. Adherence to medication is related to people's knowledge and beliefs about their disorder and illness, motivation to manage it, confidence in their ability to engage in illness-management behaviours and expectations regarding the outcome of treatment and the consequences of poor adherence (World Health Organization, 2003). It is critical and important to recognize that a person may have underlying and multiple risk factors for medication non-adherence. Also, factors that can influence a person's medication-taking behaviour may change over time to time. Therefore, it is important to continually assess and evaluate a person's adherence throughout the course and duration of therapy. In addition, because there is usually no single reason for medication nonadherence, there can be no "one size fits all" approach to improving adherence. $^{20}$

Based on published studies, it is proven with evident that single interventions are less successful than multiple, long-term interventions in affecting adherence. Studies have shown that the most successful interventions have some follow-up component and address the underlying reason(s) for non-adherence Krueger et al. 2003. Comprehensive and complete interventions should address and emphasize a variety of issues, including knowledge of the treatment, motivation form patient, social support and individualizing therapy based on a person's concerns and needs Krueger et al. 2003 McDonald et al. 2002. The ideal and best time to start or initiate adherence interventions is when therapy first begins. Interventions that are initiated early in the course of therapy can support older persons through a period when they are most likely to have questions or to experience side effects from therapy.

\section{Patient-Related Factors}

Several patient-related factors, including lack of understanding of their disease and its complication, lack of involvement in the treatment decision-making process and suboptimal and insufficient medical literacy, contribute to medication non-adherence. In the United States alone, an estimated 90 million adults have inadequate and insufficient health literacy, placing them at risk for increased and raised rates of hospitalization and poorer clinical outcomes. The patient's health beliefs towards therapy and attitudes concerning the effectiveness of the treatment, their previous experiences with pharmacological therapies and disease and lack of motivation also affect the degree of medication adherence. ${ }^{21}$

\section{Physician-Related Factors}

Not only do physicians often fail to recognize and trace medication nonadherence in their patients, they may also contribute to it by giving and prescribing complex drug regimens, communication barrier like failing to explain the benefits and adverse effects of a medication effectively and inadequately considering the financial burden to the patient prior to management of drug therapy. Ineffective communication between the primary care physician and the patient or also relatives with a chronic disease such as CVD further compromises the patient's understanding of his or her disease, its potential complications and the importance of medication adherence. Failing to elicit and rule out a history of alternative, herbal, or supplemental therapies from patients is another source of ineffective communication. ${ }^{22}$

\section{Health System / Team Building-Related Factors}

Fragmented health care systems can lead to barriers to medication adherence by limiting the health care coordination and the patient's access to care treatment. Prohibitive drug costs or co-payments also contribute to poor medication adherence. Besides, health information technology is not widely available and up-to-date, preventing physicians from easily accessing information from different patient care-related venues, which in turn compromises patient care, timely medication refills and patient-physician communication. Other than that, in an overtaxed health care system in which clinicians see a large numbers and volume of patients without resources to meet individual patient needs, the amount and duration of time a clinician spends with patients may be insufficient to properly counsel and assess and understand their medication-taking behaviours. This lack of time may prevent and preclude engaging the patient in a discussion on the importance and significant of medication adherence and strategies to achieve success of their therapy. ${ }^{23}$

\section{Strategies to Improve Adherence}

Adherence is one of the important part in patients' therapy management. If we can ensure patients' adherence, we can figure out other possible factor if the treatment fail for example under dose of medication or the patients need to change regimen therapy. There are a few ways to improve patients' adherence.

\section{Patient-Related Factors}

First, we can do something that is related to the patients themselves. The psychological risk factor such as poor insight, denial of illness, negative attitude towards medication and lack of conviction that medication will pre- 
vent relapse of disease can have impact on patients' adherence to medication. This is related to patients' belief in the healthcare system.

Psych education is one of the intervention that can be used to improve patients' adherence. This intervention involves individual or group counselling session with the use of audio-visual materials on diagnosis, medications and side effects. Psychoeducation alone will not be sufficient to improve rates of medication adherence. We need to combine it with other method. Cognitive-Behavioural Therapy also can be implemented in improving patients' adherence. In this model, adherence is viewed as a coping behaviour based on the perception of the disease and the belief on the medications. ${ }^{24}$

This therapy challenge patients' thought on medications by helping patients' mentally link medication adherence to symptoms reduction and personal health. This therapy has been proved to improved adherence among patients especially with psychiatric disorder. The approaches to improve patients' adherence in his therapy including rewards, cues, reminder and skill training. This therapy aims to promote, modify and reinforce behaviours related to adherence in patients.

Next thing that we can do that is related to the patient is motivational interviewing. ${ }^{25}$ It is a patient-centered directive method to enhance intrinsic motivation to change by exploring and resolving any doubt in therapy. It was used successfully in treating addiction and can be applied to assess patients' level of motivation to adopt medication-adherence behaviours. There are stages in change which is pre-contemplation, contemplation, preparation, action and maintenance.

We have to determine the stages which our patient is in. Patients in the contemplation stage are thinking of changing but are not yet fully dedicated to changing. They aware that non-adherence can be problematic to their health status. Patients in the preparation stage are intending to adhere to their medications regularly but may have several barriers preventing them from doing so. Patients who are acting are actively working to take their medications regularly, while patients in the maintenance stage are doing so on a consistent basis. ${ }^{26}$

By using this method, clinician can tailor patients' therapy to the current level of motivation level of the patients. Studies shows that even there is decrease in effectiveness at 6-month follow up, but the adherence level in the group with this motivational interviewing intervention remain significantly higher than group without it.

Another aspect that is important to improve patients' adherence is the communications with patients. Only about $50 \%$ of patient going home not knowing what they have been told by the clinicians. And 54\% of patients' problem and $45 \%$ of patients' concern are neither elicited by the clinician nor disclosed by the patient. The problems and concerns might relate to their medical condition and hindering them to adhere to their medication as the clinician cannot tailor the therapy accordingly. Some research shows that $71 \%$ of patients stated poor relationship as a reason for their malpractice claims. To overcome this barrier, we can built a good patients-practitioners relationship.

The assessment of patients' motivation should be done prior to start a treatment. The unmotivated patients will be less likely to start and maintain the recommended treatment. It is recommended for clinicians to directly asking the patient and discuss about patients' motivation at the beginning like 'How motivated are you take your medication?' or question similar like this. After that, clinicians can encourage patient to discuss the pros and cons of taking the medications as prescribed. Patients also should be prompted to speak their own intent to change in order to increase the probability that they will adopt the behaviour necessary to change. Clinicians can help patients to make a relationship between the benefits of taking medicine and symptoms reduction. Then, after patient already make step towards behaviour change, the clinician should give positive feedback to reinforce the behaviours. ${ }^{27}$

There are ways to provide information to patients. Firstly, the instruction should be limited to 3 or 4 major point during each discussion. Secondly, using simple language when explaining the disease and when giving instructions. Thirdly, by giving written material such as pamphlet during oral teaching. Fourthly, involving patients' family members or friends. Lastly, reinforce the concept discussed during each appointment. Eleven study that using education-based type of intervention was analysed in a meta-analysis study by J.A Rash et al. to improved patients' adherence. Three out of the eleven studies reported improvement in adherence with the intervention by using provision of four risk factor counselling session, compare to usual care. ${ }^{26} \mathrm{But}$, there are also several education-based interventions that are not successful. This include shared-care guidelines, one risk-factor education session, additional electronic information to nurse management, written education combined with brief medication adherence session and more intensive provision follow up education delivered by pharmacists or counsellor. We can modify this kind of education-based intervention that may suit with the patient because it was found to have no influence on patients' adherence. 


\section{Therapy-Related Factors}

There are many therapy regimes for a single medical condition. The therapy chose by the clinical might affect patients' adherence to medication. Studies shows that patient who undergo individualized disease management intervention have better compliance compare to patients treated as usual. ${ }^{27}$

For example, patients were given a diary to record anything regarding their medical condition and during appointment with clinician, they discuss whatever medical condition that is written in the diary such as the effectiveness of inhaler in asthma patient. This method significantly increases patients' adherence towards medications. Other study also shows that the regimen that use less frequency of medication that need to be taken by the patients improving adherence to medications.

For example, in Highly Active Anti-Retroviral Therapy (HAART). There is improvement in adherence in once daily regimen compared to twice daily regimen. Through this study, we know that one of the effective method is to simplify the regimen of the therapy. ${ }^{24}$

Simplifying the regimen does not mean to change the less frequency of the medication only that can be done with a longer acting medications. For example, changing captopril which is usually taken three times daily to perindopril which only need to be taken once daily. Sometimes, there is no other option to change the frequency of the medication because of the availability of the medication. If this happened, we can try to match the regimen to match to patients' activities of daily living. People are more likely to remember to take medications before a meal or before going to sleep..$^{28}$

The interpretation of the labels on how they should be taking the medications also important. Study by Eraker et al. found that only $36 \%$ of patients correctly interpreted the meaning of the instruction of 'every 6 h'. Unless we have no other option in the regimen therapy, we should simplify it in order to improve patients' adherence to medications. Another metaanalysis study by J.A Rash et al. found that adherence can be improved by simplification of drug regimen. There are three study (Castellno et al. 2014, Patel et al. 2015 and Thorn et al. 2013) test the intervention of simplifying drug regime by using a polypill. It is basically combine statin therapy with other indicated antihypertensive agents. ${ }^{29}$

\section{Social and Economic Factors}

Thirdly, another factor that could influence patients' adherence to medications is the social and economic factor. Socioeconomic status seems not consistently to be the predictor of adherence. However, in developing countries, patients with low socioeconomic status may in the position of having to choose between priorities. Some other factors that also have significant effect on adherence are including poverty, low level of education, unemployment, lack of effective social support networks, unstable living conditions, long distance from treatment centre, high cost of transport, high cost of medicine and changing environmental situation. In this kind of situation, there is not much that the staff at the clinic and hospital can do. High cost of medicine is reported as an important cause of non-adherence in developing countries. It is suggested that the policy maker to design and manage healthcare environment needs. For example, Malaysian National Medicine Policy to ensure the availability and affordability of the product. ${ }^{30}$

Malaysian citizen can have choice whether they want to go to private or government health facility. For patients' who is in low economic status, they have choice to go to the government health facility that is affordable for everyone. We can also promote the use of generic medications which is less expensive than the innovator brands. By having these, we can ensure that all patients have equal chance to get their medications thus reduce the likelihood of non-adherence because of the availability and affordability of the medications.

Community-based organization and social support group have been reported to be effective to improve adherence and behaviours. It is also has been reported to improve adherence to prescribed medications for treating chronic disease such as diabetes, epilepsy, asthma and hypertension. The elements that are needed in the community-based programs is to promote the exchange of the experience of handling the disease and the treatment; providing comprehensive medical information and also promoting each member of the group's responsibility for their own care. ${ }^{31}$

Another approach that is suitable for patients with low economic status is by providing financial incentives to them. In a study where patients receive $\$ 22$ financial incentive for each injection received for over 12 months, the adherence increase from $69 \%$ at baseline to $85 \%$ after the 12 months treatment. Furthermore, the percentage of individual patients achieve more than or equal to $95 \%$ adherence increase from $7 \%$ to $28 \%$ compare to increase from $2 \%$ to $5 \%$ in individual patients who did not receive any financial incentive. Again, the use of this approach must be accompanied by other method to increase patients' adherence.

A meta-analysis study by J.A Rash et al. found that there is improvement in medication adherence with the intervention of prescription cost coverage. But, the effect size is small because only one study by Choudry et al. 
that test this kind of intervention in patients' adherence towards medication. More study needed to know the real effect of the intervention. However, we can include this in treatment plan for patient who is not adhere to the treatment plan.

\section{Health Care Team and System-Related Factors}

Fourthly, we can adjust the health care system and also do some intervention with the health care team. There is no single method has been shown to be effective across all patients. The intervention that that target adherence must be tailored to the illness-related demands experienced by the patients.

To achieve this, health systems and the provider needs to develop something that accurately assessing not only adherence, but also the factor to contribute it as the healthcare provider are expected to play their important role in promoting adherence. To practice this, practitioners must undergo specific training in adherence management. The training should cover three topics simultaneously. ${ }^{32}$

First, the practitioner need to have the information about adherence such as the factors that is reported to affect adherence, the available affective intervention at the health facility and the epidemiology and economics of adherence and behavioural mechanism that can promote patientrelated adherence. Second, how practitioners can make use of the information to improve patients' adherence. This should include the assessment tools and strategies to promote change in patients' adherence. Practitioners should be trained to assess the level of adherence of their patients and do some intervention accordingly. Third, they should know how to use behavioural tools to create or if the adherence of the patient is satisfactory, to maintain that adherence habit.

Research by Marder et al. found that patients who were adhere to their medications were more likely to be satisfied with their practitioners, felt that the practitioners understand them and the practitioner had their best interest in mind. This alliance can also be facilitated to be positive by identifying the treatment goal and the practitioners must ally themselves with the healthy aspects of the patients' ego that are striving to reach the goals. The collaboration between the patient and practitioners must identify the mutually agreed upon treatment goals and strategies including medications and the patients must be viewed as the stakeholder in the treatment plan. ${ }^{33}$

\section{Adherence Counselling}

Patient counselling is a very important for healthcare professional especially pharmacists and it should be part of pharmacists training. To optimize the counselling, healthcare professional need to tailor patients need with the desired outcome. To conduct a good counselling session, one should have the skill of listening, questioning, respect and negotiation. It should be a twoway interactive process between patients and the healthcare provider where the healthcare provider will try to trigger something that will make the patients to respond and seek further information required. The aim of the counselling is to understand the needs of the patients and solving the problem with patients' no-adherence problem and also maintaining quality of health and quality of life. ${ }^{33,34}$

Adherence to medication important for patient health progression especially for medication to counter infection in patients' body like HIV/AIDS. Adherence is the second strongest progression to AIDS. The usual cause for treatment failure is because of poor adherence. ${ }^{32}$

\section{Steps in Counselling for Treatment Preparation and Adherence}

The first step is to establish the relationship and rapport with the patient. We can give any necessary information and guidance to the patient. We tell the objective of the treatment. For example, patient with diabetes. If the healthcare team want to start insulin therapy, we need to tell the patient the indication and importance of taking insulin as directed. We can also identify treatment support group for the patient and encourage involvement of family members or friends of the patient. From this, we can assess the sociological background and support that the patient have. From the interview with the patient also, we can know patients lifestyle and daily events. It is important to tally and individualized the treatment based on patient's lifestyle unless it will not be very successful. After knowing background of the patient, the readiness of the patient need to be assessed. We can assess patient's readiness by looking at the ability of the patient to attend clinic regularly and do not miss appointment or if there is any record that patient able to complete full course of antibiotic if available. ${ }^{35}$

For some treatment like antiretroviral therapy, the adherence need to as high as $95 \%$ to avoid resistance to the medications which means missing dose should be less than three times. For antiretroviral therapy, patient should be able to adhere $100 \%$ to the medication. We need to assess patient's adherence regularly. ${ }^{36-38}$ If the patient has difficulty to adhere the medication, we need to reinforce the adherence counselling and list out the barriers again to develop strategies to overcome it. ${ }^{39}$ 
Other than that, we also need to explain the possible side effect of the medication and the method to counter it so that patient can prepare it if it happens in the future and not feel shock with the side effect. Possible interaction of the medication also need to be explained to the patient including drug-drug interaction, drugfood interaction and also drug-herb interaction. For example, if we want to start warfarin treatment to the patient, we need to advise about food intake especially. ${ }^{40}$ The second step is to conduct the counselling after establishing the relationship in one or more individual session. During this session, we need to know patient's feeling about the whole treatment plan. Everyone has their own problem that might be related to family, job, relationships and others kind of problems. We cannot focus to have strict adherence until these negative feelings of the problem are sorted out. Many patients do not have a special and private place to store the medication. Some do not want others to know their disease status like HIV/AIDS patients. ${ }^{41,42}$

Other than that, we need to check for any financial difficulties that the patient experienced. Certain patient, especially the one who has lower socioeconomic status and live in rural area, may have problem to travel to the health facility or the health problem was affected by a poor diet. All this factor need to be into the consideration during counselling.

The third step is to solve the problems that the patient experienced and create a treatment plan. In the second step, we already determined patient's problem and barriers to adherence (also discussed in previous chapter). ${ }^{42}$ We need to tally and solve the problem that create barriers to adherence. For example, if the patient is on captopril medication for hypertension that is needing to be taken three times a day. Patient's job nature is to always travel and do not have time to take the second and the third dose and always complain cannot take the second and the third dose on time every day. ${ }^{43}$ We may want to address the problem to the prescriber and suggest changing the regime to perindopril that only need to be taken once daily. Some of the key words that we need to highlight during counselling are:

a) Missing dose can cause relapse and resistance (for example antibiotic).

b) If patient missed the dose, the patient need to take the dose as soon as possible as long as it is not near the time for next dose and not to double the dose.

c) Always check the expiry date of the medication. Do not take the expired medication. For durable insulin pen, do not take the insulin if the insulin is already in the pen for one month. d) Patient must not share the medication with others including friends and families.

e) Always discuss with the healthcare provider if the patient having difficulties in the treatment plan.

We need to remember that counselling is not only to increase patient's knowledge and adherence, it also makes an improvement in the quality of care. Various studies show that informed group of patients with knowledge and potential side effect have better adherence and safety. Counselling is a method to facilitate rational and adherence behaviour of the patient. Some of the techniques to assist patient for adherence are:

a) Maintaining the good relationship with patient

b) Emphasize the key point

c) Give reason for any advice that we provide

d) Give concrete and definite instructions

e) Supply the patient with counselling written aid like pamphlet during counselling session

f) Always ensure feedback from the patient

During counselling session, we need to assess patient's adherence by using method explained in the previous chapter. ${ }^{44}$

\section{CONCLUSION}

Patient medication non-adherence is a major and medical problem and phenomenon globally. There are many inter related reasons and causes for the same. Though patient education level is the key to improving compliance, use of compliance aids, proper motivation by health care provider and support is also shown to increase medication adherence. Health care professionals should identify practically possible strategies to improve and aid medication adherence within the limits of their practice eventually enhancing therapeutic outcome. It should be a combination of multidisciplinary approach that needs to be carried out with the support of all those who are involved in medication use. Poor and medication adherence is a key hindrance in combating the challenges of public health in both developed and developing countries. For successful pharmacotherapy, healthcare professionals and researchers should utilize and practice all available methods within their limits of practice to improve and enhance the medication adherence.

\section{ACKNOWLEDGEMENT}

We would like to dedicate our acknowledgement of gratitude towards to Dr. Santosh Fattepur, Head of Pharmacology unit, School of Pharmacy, Management and Science University for his support in this publication. 


\section{CONFLICT OF INTEREST}

The authors declare no conflict of interest.

\section{ABBREVIATIONS}

MPR: Medication Possession Ratio; MEMS: Medication event monitor; CHARM: Candesartan in Heart Failure: Assessment of Reduction in Mortality and Morbidity; LDL: Low Density Lipoprotein; CAMIAT: Canadian Amiodarone Myocardial Infarction Arrhythmia Trial; CVD: Cardiovascular disease; HAART: Highly Active Anti-Retroviral Therapy.

\section{REFERENCES}

1. Selak V, Elley CR, Crengle S, et al. Improving adherence using combination therapy (IMPACT): Design and protocol of a randomised controlled trial in primary care. Contemp Clin Trials. 2011;32(6):909-15. doi: 10.1016/j. cct.2011.07.006.

2. Carter BL, Levy BT, Gryzlak B, et al. A centralized cardiovascular risk service to improve guideline adherence in private primary care offices. Contemp Clin Trials. 2015;43:25-32. doi: 10.1016/j.cct.2015.04.014.

3. El-Mallakh P, Findlay J. Strategies to improve medication adherence in patients with schizophrenia: The role of support services. Neuropsychiatr Dis Treat. 2015;11:1077-90. doi:10.2147/NDT.S56107.

4. Atreja A, Bellam N, Levy SR. Strategies to enhance patient adherence: Making it simple. Med Gen Med. 2005;7(1):4. doi: http://dx.doi.org/10.2147/ RMHP.S19801.

5. Julius RJ, Novitsky MA, Dubin WR. Medication adherence: A review of the literature and implications for clinical practice. J Psychiatr Pract. 2009;15(1):34-44. http://ovidsp.ovid.com/ovidweb. cgi?T=JS\&PAGE=reference\&D=emed9\&NEWS=N\&AN=2009350119.

6. Farris KB, Salgado TM, Batra $\mathrm{P}$, et al. Confirming the theoretical structure of expert-developed text messages to improve adherence to anti-hypertensive medications. Res Soc Adm Pharm. 2016;12(4):578-91. doi: 10.1016/j. sapharm.2015.09.009

7. Medication R. To Improve Medication Adherence. Pharm Pract. 2003;60(7):657-65. http://www.medscape.com/viewarticle/452568_ References.

8. Julie K, Record EJ, Palmer-Bacon J. A review of behavioural tailoring strategies for improving medication adherence in serious mental illness. Dialogues in Clinical Neuroscience. 2012;319-51.

9. Bidwal M, Lor K, Yu J, Ip E. Evaluation of asthma medication adherence rates and strategies to improve adherence in the underserved population at a Federally Qualified Health Center. Res Social Adm Pharm. 2017;13(4):75966. doi: 10.1016/j.sapharm.2016.07.007.

10. Rash JA, Campbell DJT, Tonelli M, Campbell TS. A systematic review of interventions to improve adherence to statin medication: What do we know about what works?. Prev Med. 2016;90:155-69. doi: 10.1016/j. ypmed.2016.07.006.

11. Lo TO. Adherence to Long-Term Therapy-Evidence for Action. World Health Organization. 2003.

12. Peterson AM, Takiya L, Finley R. Meta-analysis of trials of interventions to improve medication adherence. Am J Heal Syst Pharm. 2003;60(7):657-65.

13. Stonerock GL, Blumenthal JA. Role of Counseling to Promote Adherence in Healthy Lifestyle Medicine: Strategies to Improve Exercise Adherence and Enhance Physical Activity. Prog Cardiovasc Dis. 2017;59(5):455-62. doi: 10.1016/j.pcad.2016.09.003.

14. Ho LY, Camejo L, Kahook MY, et al. Effect of audible and visual reminders on adherence in glaucoma patients using a commercially available dosing aid. Clin Ophthalmol. 2008;2(4):769-72.

15. Charles T, Quinn D, Weatherall $M$, et al. An audiovisual reminder function improves adherence with inhaled corticosteroid therapy in asthma. J Allergy Clin Immunol. 2007;119(4):811-6.
16. Vervloet M, Linn AJ, Weert JCMV, et al. The effectiveness of interventions using electronic reminders to improve adherence to chronic medication: A systematic review of the literature. J Am Med Informatics Assoc. 2012;19(5):200-9. doi: 10.1136/amiajnl-2011-000748.

17. MacDonald L, Chapman S, Syrett M, Bowskill R, Horne R. Improving medication adherence in bipolar disorder: A systematic review and metaanalysis of 30 years of intervention trials. J Affect Disord. 2016;194:202-21. doi: 10.1016/j.jad.2016.01.002

18. Rash JA, Lavoie KL, Sigal RJ, et al. The OPTIMIZE trial: Rationale and design of a randomized controlled trial of motivational enhancement therapy to improve adherence to statin medication. Contemp Clin Trials. 2016;49:4756. doi: 10.1016/j.cct.2016.06.001.

19. $\mathrm{Rb} \mathrm{H}$, Ackloo $\mathrm{E}$, Sahota $\mathrm{N}$, et al. Interventions for enhancing medication adherence (Review) Interventions for enhancing medication adherence. Cochrane database Syst Rev. 2008;2(2):CD000011. doi:10.1002/14651858.

20. Lam WY, Fresco P, Lam WY, Fresco P. Medication Adherence Measures: An Overview, Medication Adherence Measures: An Overview. Bio Med Res Int. 2015;e217047. doi:10.1155/2015/217047, 10.1155/2015/217047.

21. Knapp M, King D, Pugner K, Lapuerta P. Non-adherence to antipsychotic medication regimens: Associations with resource use and costs. $\mathrm{Br} \mathrm{J}$ Psychiatry. 2004;184(6):509-16. doi:10.1192/bjp.184.6.509.

22. Lippman A. Adherence to medication. N Engl J Med. 2005;353(18):4. http:// www.ncbi.nlm.nih.gov/pubmed/16270426.

23. Sokol MC, McGuigan KA, Verbrugge RR, Epstein RS. Impact of medication adherence on hospitalization risk and healthcare cost. Med Care. 2005;43(6):521-30. doi: 10.1097/01.mlr.0000163641. 86870.af.

24. Hong JS, Kang HC. Relationship between oral antihyperglycemic medication adherence and hospitalization, mortality and healthcare costs in adult ambulatory care patients with type 2 diabetes in South Korea. Med Care. 2011;49(4):378-84. doi:10.1097/MLR.0b013e31820292d1.

25. Moral RR, DeTorres LAP, Ortega LP, et al. Effectiveness of motivational interviewing to improve therapeutic adherence in patients over 65 years old with chronic diseases: A cluster randomized clinical trial in primary care. Patient Educ Couns. 2015;98(8):977-83. doi: 10.1016/j.pec.2015.03.008.

26. Bender BG, Rand C. Medication non-adherence and asthma treatment cost. Curr Opin Allergy Clin Immunol. 2004;4(3):191-5. doi: 10.1097/00130832200406000-00009.

27. Kane S, Shaya F. Medication non-adherence is associated with increased medical health care costs. Dig Dis Sci. 2008;53(4):1020-4. doi: 10.1007/ s10620-007-9968-0.

28. Hou MY, Hurwitz S, Kavanagh E, et al. Using daily text-message reminders to improve adherence with oral contraceptives: A randomized controlled trial. Obstet Gynecol. 2010;116(3):633-40.

29. DaCosta FA, Guerreiro JP, DeMelo MN, et al. Effect of reminder cards on compliance with antihypertensive medication. Int $\mathrm{J}$ Pharm Pract. 2005;13(3):205-11.

30. Saleh F, Mumu SJ, Ara F, Hafez MA, Ali L. Non-adherence to self-care practices and medication and health related quality of life among patients with type 2 diabetes: A cross-sectional study. BMC Public Health. 2014;14(1):431. doi: 10.1186/1471-2458-14-431.

31. Srikanth BA, Purushothama KR, Narotham KR, et al. Patient counselling tools for improving adherence to antiretroviral therapy. Int J Pharm Sci Res. 2012;3(12):4634-9. http://www.embase.com/ search/results? subaction=viewrecord\&from=export\&id=L368401263। nhttp://www.ijpsr.com/V3I12/10 Vol. 3, Issue 12, December 2012, IJPSR, RE 773, Paper10.pdfinhttp://rug.on.worldcat.org/atoztitles/ link/?sid=EMBASE\&issn=23205148\&id=doi:\&ati.

32. Figaji AA, Ch MBB. Adherence to HIV Treatment Regimens: Recommendations for Best Practices. Guidelines for the use of Antiretroviral Agents in Adults and Adolescent with HIV. 2008;63(1):83-92. http://aidsinfo. nih.gov/guidelines. doi: 10.1227/01.NEU.0000313113.43447.0C.

33. Hardy H, Kumar V, Doros $\mathrm{G}$, et al. Randomized controlled trial of a personalized cellular phone reminder system to Enhance adherence to antiretroviral therapy. AIDS Patient Care STDS. 2011;25(3):153-61.

34. Andrade AS, McGruder HF, Wu AW, et al. A programmable prompting device improves adherence to highly active antiretroviral therapy in HIV-infected subjects with memory impairment. Clin Infect Dis. 2005;41(6):875-82. 
35. Pop-Eleches $\mathrm{C}$, Thirumurthy $\mathrm{H}$, Habyarimana JP, et al. Mobile phone technologies improve adherence to antiretroviral treatment in a resourcelimited setting: A randomized controlled trial of text message reminders. AIDS. 2011;25(6):825-34.

36. Simoni JM, Huh D, Frick PA, et al. Peer support and pager messaging to promote antiretroviral modifying therapy in settle. A randomized controlled trial. J Acquire Immune Defic Syndr. 2009;52(4):465-73.

37. Simoni JM, Chen WT, Huh D, et al. A preliminary randomized controlled trial of a nurse-delivered medication adherence intervention among HIV-positive outpatients initiating antiretroviral therapy in Beijing, China. AIDS Behav. 2011;15(5):919-29.

38. Safren SA, Hendriksen ES, Desousa N, et al. Use of an on-line pager system to increase adherence to antiretroviral medications. AIDS Care. 2003;15(6):787-93.

39. Christensen A, Christrup LL, Fabricius PE, et al. The impact of an electronic monitoring and reminder device on patient compliance with antihypertensive therapy: A randomized controlled trial. J Hypertens. 2010;28(1):194-200.
40. Christensen A, Christrup LL, Fabricius PE, et al. Survey of patient and physician assessment of a compliance reminder device in the treatment of hypertension. Blood Press. 2009;18(5):280-5.

41. Santschi V, Wuerzner G, Schneider MP, et al. Clinical evaluation of IDAS II, a new electronic device enabling drug adherence monitoring. Eur J Clin Pharmacol. 2007;63(12):1179-84.

42. Strandbygaard $U$, Thomsen SF, Backer V. A daily SMS reminder increases adherence to asthma treatment: A three-month follow-up study. Respir Med. 2010;104(2):166-71.

43. Laster SF, Martin JL, Fleming JB. The effect of a medication alarm device on patient compliance with topical pilocarpine. J Am Optom Assoc. 1996;67(11):654-8.

44. Eraker SA, Kirscht JP, Becker MH. Understanding and improving patient compliance. Ann Intern Med. 1984;100(2):258-68.

\section{About Authors}

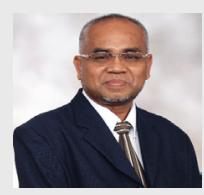

Dr. Yahaya Hassan, is a Professor of Clinical Pharmacy at School of Pharmacy, Management and Science University (MSU), Malaysia. His current research interest focus on Clinical Pharmacy, Pharmacoepidemiology and Pharmaceutical outcome study. He also, serve as a consultant for Malaysian Qualifying Agentcy (MQA) Ministry of Higher Education of Malaysia and MQA panel member for Board of Pharmacy of Malaysia. He also serve as a committee member of Clinical Pharmacy Specialist development program for Pharmacy Division, Ministry of Health of Malaysia. $\mathrm{He}$ has been lecturing at the faculty of Pharmacy for more than 35 years covering courses in Clinical Pharmacy, Pharmacokinetics, Advanced Pharmacotherapeutics and Pharmacy practice. He has published over 200 research papers in international journals.

Cite this article: Hassan Y, Al-Temimi AA, Ramli R, Saad MFM, Aziz NA. Difficulties Facing the Patients and Extra Burden for Healthcare Providers by Non-adherence to the Medications - A Comprehensive Review. Indian J of Pharmaceutical Education and Research. 2019;53(4s):s487-s499. 